\title{
Distributed antenna selection with Message Passing Algorithm for MIMO D2D communications
}

\author{
Yi Yu, Mylene Pischella and Didier Le Ruyet \\ CNAM CEDRIC \\ 292 rue Saint-Martin - 75003 Paris, France \\ Email: yi.yu.eicnam@gmail.com; mylene.pischella@cnam.fr; didier.le_ruyet@cnam.fr;
}

\begin{abstract}
This paper presents two algorithms for optimizing the transmit and receive antenna selection matrices in Multiple Input Multiple Output (MIMO) Device-to-Device (D2D) communications. MIMO antenna selection technique is well-suited for D2D communications since it is relevant to low-cost devices with less Radio Frequency (RF) chains than antennas, and requires low feedback. The proposed algorithms determine the optimal antenna selection matrices, taking into account both direct and interfering MIMO channels, with the distributed MessagePassing (MP) algorithm. A joint and an iterative algorithm are proposed and assessed. The joint algorithm provides large data rates, while the low-complexity iterative algorithm is more efficient than a reference individual transmit antenna selection ignoring interference.
\end{abstract}

\section{INTRODUCTION}

Device-to-Device (D2D) communications can be established between two nearby mobile users that are currently exchanging data. Such a direct communication decreases the latency, increases the data rate and decreases the required power consumption. D2D pairs may be reusing the spectrum if they generate low interference to each others [1]. Interference can be handled through power control [2]. In Multiple Input, Multiple Output (MIMO) transmissions, antenna selection [3], [4] is a low-feedback technique to perform stream selection by using a subset of the available antennas. This technique is also cost-efficient and well-adapted to the D2D context since the number of required transmit-receive Radio Frequency (RF) chains per device is then lower than the number of transmitreceive antennas. In this paper, we optimize antenna selection at both transmitters and receivers to maximize the sum rate of D2D pairs, while taking into account interference. Two distributed algorithms are obtained by using the MessagePassing algorithm (MPA).

Factor graphs (FG) and the associated MPA have been used to efficiently solve different problems in communications including the decoding of low-density parity check (LDPC) codes, turbo codes and turbo equalization [5] [6]. A FG is an undirected bipartite graph that describes the factorization of multivariate functions. It connects variables and factors where each factor represents a function over the variables it is connected to. FG and MPA provide an efficient way to compute functions involving a large number of variables when the functions can be factorized into terms that involve only a subset of the variables. MPA can be formulated for a variety of problems requiring computations on a commutative semiring. Depending on the choice of the semiring and the associated operations (sum, product or minimum), different MPA are available such as the belief-propagation approach of maximum likelihood decoding.

Message-passing algorithms have also been considered to solve optimization problems [7]. In [8], the authors have applied the sum-product algorithm to a function related to the sum-rate for the downlink transmit beamforming problem in a multi-cell MIMO system. In [9], different MPA are used to perform distributed downlink beamforming for cooperative base stations.

Recently, Guerreiro and al. [10] have proposed a method based on the min-sum algorithm applied to the problems of transmit antenna selection and fixed-beam selection sum-rate function. This work was extended to the optimization of a multicell network where each cell needs to select precoding matrices for beamforming in a distributed way in [11]. In [12], the authors introduce also a method based on message-passing formulation to solve the problem of interference alignment (IA) over MIMO channels.

In this paper, we propose to solve the problem of selecting antenna at transmitters and receivers with MPA. A joint transmit-receive antenna selection algorithm is first proposed, and then a lower-complexity iterative transmit and receive antenna selection algorithm is derived. These algorithms are fully distributed since they rely on MPA. Consequently, they could be implemented in future $5 \mathrm{G}$ distributed D2D communications networks, where the Base Station (BS) does not serve as the controller.

The paper is organized as follows. In section II, the D2D communications system model is detailed and MPA for resource allocation is presented. Section III provides two algorithms for antenna selection in MIMO D2D communications. Their performance results are evaluated in section IV, and section $\mathrm{V}$ concludes the paper.

\section{SYSTEM MODEL}

\section{A. MIMO D2D system model}

We consider $K$ D2D pairs located within a circular area of radius $r$. Each D2D receiver's location is uniformly distributed within $r_{0}$ to $r_{D}$ from its transmitter's location. D2D transmitters are all active in the same channel of bandwidth $B$. All transmit and receive devices are equipped with $N_{t}$ and $N_{r}$ multiple antennas, respectively. Antenna selection is 
performed both at transmitters and at receivers. Only $N_{s}$ antennas are selected at transmitters and receivers. To simplify notations, we refer to $j$ for both transmitter and receiver of D2D pair $j . \mathcal{S}_{j}$ is defined as the set of D2D pairs that interfere D2D receiver $j$. It is equal to the set of D2D pairs $k$ whose transmitter has a distance to the receiver of D2D pair $j$ that is lower than a threshold, called $D_{0}$, with the exception of the transmitter of D2D pair $j$. We assume that the interference generated at receiver $j$ by transmitters with a higher distance can be neglected. This assumption is taken in order to decrease the antenna selection algorithm's complexity.

Let $\mathbf{H}_{j k} \in \mathbf{C}^{N_{r} \times N_{t}}$ be the MIMO channel response, including only multi-path fading, from transmitter $k$ to receiver $j$, with $(k, j) \in\{1, . ., K\}$. To simplify notations, the channel response from the transmitter to the receiver of the same D2D pair $k$ is noted $\mathbf{H}_{k k}$. Let $\mathbf{s}_{k} \in \mathbf{C}^{N_{s} \times 1}$ be the stream vector of D2D transmitter $k$ and $\mathbf{V}_{k} \in \mathbf{C}^{N_{t} \times N_{s}}$ be the precoding matrix of transmitter $k$. At each receiver $j$, a receive matrix $\mathbf{W}_{j} \in \mathbf{C}^{N_{r} \times N_{s}}$ is applied on the received signal $\mathbf{y}_{j} \in \mathbf{C}^{N_{r} \times 1}$ as follows: $\widehat{\mathbf{s}_{j}}=\mathbf{W}_{j}^{H} \mathbf{y}_{j}$. The transmit power of transmitter $k$ is noted $\phi_{k}$ and the inverse of path loss and shadowing from transmitter $k$ to receiver $j$ is noted $g_{j k}$. Then the data rate at receiver $j$ is:

$$
\begin{aligned}
& R_{j}= \\
& B \log _{2}\left(\operatorname{det}\left(\mathbf{I}_{N_{r}}+g_{j j} \frac{\phi_{j}}{N_{s}} \mathbf{Q}_{j}^{-1} \mathbf{W}_{j}^{H} \mathbf{H}_{j j} \mathbf{V}_{j} \mathbf{V}_{j}^{H} \mathbf{H}_{j j}^{H} \mathbf{W}_{j}\right)\right)
\end{aligned}
$$

where $\mathbf{I}_{N_{r}}$ is the identity matrix of size $N_{r}$, and $\mathbf{Q}_{j}$ is the covariance matrix of noise plus interference at receiver $j$, defined as:

$$
\mathbf{Q}_{j}=\mathbf{W}_{j}^{H}\left(\mathbf{N}_{q}+\sum_{k \in \mathcal{S}_{j}} g_{j k} \frac{\phi_{k}}{N_{s}} \mathbf{H}_{j k} \mathbf{V}_{k} \mathbf{V}_{k}^{H} \mathbf{H}_{j k}^{H}\right) \mathbf{W}_{j}
$$

$\mathbf{N}_{q}$ is the noise covariance matrix, equal to $N_{0} B \mathbf{I}_{N_{s}}$ for white noise with power spectral density $N_{0}$.

The transmit and receive matrices can be optimized to maximize the sum data rate. In order to have low-cost devices and low feedback requirements, transmit and receive matrices are antenna selection matrices [3]. In D2D communications with interfering D2D pairs, sum rate maximization is achieved not only be exploiting the best direct channel directions, but also by avoiding the most interfered ones. In the following, we propose two algorithms to determine the best set of matrices $\mathbf{V}_{k}, k \in\{1, . ., K\}$ and $\mathbf{W}_{k}, k \in\{1, . ., K\}$, chosen among the set of antenna selection matrices. For instance, if $N_{t}=N_{r}=3$ and $N_{s}=2$, the set of receive antenna selection matrices $\mathcal{W}$ and the set of transmit antenna selection matrices $\mathcal{V}$ are:

$$
\mathcal{W}=\mathcal{V}=\left\{\left(\begin{array}{ll}
1 & 0 \\
0 & 1 \\
0 & 0
\end{array}\right),\left(\begin{array}{ll}
1 & 0 \\
0 & 0 \\
0 & 1
\end{array}\right),\left(\begin{array}{ll}
0 & 0 \\
1 & 0 \\
0 & 1
\end{array}\right)\right\}
$$

Please notice that power allocation is not optimized and power is equally shared on all selected antennas. Including power allocation in the problem is a future research direction.
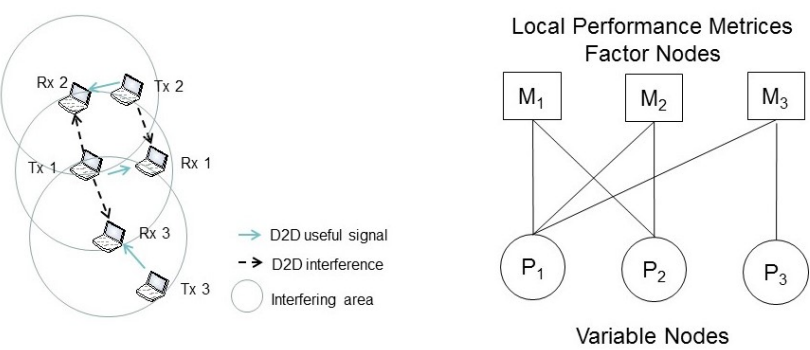

Fig. 1. An example of a 3 D2D pairs communication network and the factor graphs of a particular case

\section{B. Message-Passing Algorithm for resource allocation}

Message-Passing algorithms like the min-sum algorithm can be applied in optimization problems such as resource allocation problems to reduce the complexity and allow for distributed implementations. $P=\left\{P_{1}, P_{2}, \ldots, P_{|\mathcal{P}|}\right\}$ is the set of variables of the optimization problem.

The min-sum algorithm can solve an optimization problem whose objective is of the form

$$
\max \sum_{i=1}^{K} R_{i}=\min \sum_{i=1}^{K}\left(-M_{i}\right)
$$

where $R_{i}=-M_{i}$ is the $i^{\text {th }}$ individual function, that depends not only on parameter $P_{i}$, but also on a subset of the other parameters $\left\{P_{k}\right\}_{0 \leq k \leq|\mathcal{P}|}$. The algorithm is distributed, as it only computes the value of $M_{i}$ at node $i . M_{i}$ and $P_{i}$ represent factor nodes and variable nodes, respectively. In the factor graph, a factor node is connected to a variable node through an edge only when the corresponding function of the factor node is dependent on that given variable.

For instance, Fig.1 provides an example of factor graph for $K=3$ D2D pairs. The interference sets are the following: $\mathcal{S}_{1}=\{2\}, \mathcal{S}_{2}=\{1\}, \mathcal{S}_{3}=\{1\} . M_{i}$ is here defined as the opposite of the data rate of user $i$, given by eq.(1). The inclusive neighbor set $\mathcal{A}_{i}$ is defined as the union of $\mathcal{S}_{i}$ and $\{i\}$. In the factor graph, factor node $i$ is connected with all the variables nodes $P_{k}$ whose index $k$ belongs to $\mathcal{A}_{i}$. As shown by Fig.1, the factor graph representing D2D communications is not fully connected and depends on the interference sets.

Factor graphs express the factorization of a multivariate function into several small local functions. With respect to the communication situation at hand, each factor node $M_{i}$ denotes the local function node with its local performance metrics $M_{i}\left(p_{A_{i}}\right)$ after the factorization of the whole communication network with the global performance metric $M(p)$. Each variable node $P_{i}$ is associated with the parameter $p_{i}$ of a communication node.

Message-passing algorithm is composed of message computation and message exchanges, performed iteratively between factor nodes and variable nodes. We use $\mu_{M_{i} \rightarrow P_{k}}$ to denote the message passed from factor node to variable node, and 
$\mu_{P_{k} \rightarrow M_{i}}$ to denote the message passed from variable node to factor node.

The min-sum algorithm applied to solve problem (4) is following the steps:

1) Initialize all messages $\mu_{P_{j} \rightarrow M_{i}}\left(P_{k}\right)$ to zero:

$$
\mu_{P_{j} \rightarrow M_{i}}\left(P_{k}\right)=0
$$

2) Message passing from factor node to variable node:

$$
\begin{aligned}
& \mu_{M_{i} \rightarrow P_{k}}\left(P_{k}\right) \\
& =\min _{p_{A_{i} \backslash\{k\}}}\left\{M_{i}\left(p_{A_{i}}\right)+\sum_{j \in A_{i} \backslash\{k\}} \mu_{P_{j} \rightarrow M_{i}}\left(P_{j}\right)\right\}
\end{aligned}
$$

Where $\backslash\{k\}$ means except the node $\mathrm{k}$, and $M_{i}\left(p_{A_{i}}\right)$ is the value of the function computed at node $i$, taking into account the parameters of the nodes in set $\mathcal{A}_{i}$.

3) Message passing from variable node to factor node:

$$
\mu_{P_{k} \rightarrow M_{i}}\left(P_{k}\right)=\sum_{j \in A_{k} \backslash\{i\}} \mu_{M_{j} \rightarrow P_{k}}\left(P_{k}\right)
$$

4) After several iterations in calculating $\mu_{M_{i} \rightarrow P_{k}}\left(P_{k}\right)$ and $\mu_{P_{k} \rightarrow M_{i}}\left(P_{k}\right)$, described in step 2) and step 3$)$, compute the optimal parameter $p_{i}^{*}$ :

$$
p_{i}^{*}=\arg \min _{p_{i}}\left\{\sum_{j \in A_{i}} \mu_{M_{j} \rightarrow P_{i}}\left(P_{i}\right)\right\}
$$

where $p_{i}^{*}$ is the final results by using the min-sum message passing algorithm in this transmit-receive antenna selection problem.

The min-sum algorithm converges when the factor graph is cycle-free. Otherwise, it still provides some strong performance results, as shown in [10]-[12].

\section{Message-Passing Algorithm for Antenna SELECTION IN D2D COMMUNICATIONS}

\section{A. Optimization problem}

The optimization problem's objective is to maximize the sum data rate by determining the best transmit and receive antenna selection matrices:

$$
\max _{\substack{\left[\mathbf{V}_{1}, \ldots, \mathbf{V}_{K}\right] \in \mathcal{V} \\\left[\mathbf{W}_{1}, \ldots, \mathbf{W}_{K}\right] \in \mathcal{W}}} \sum_{j=1}^{K} R_{j}
$$

Since this problem is highly complex to be solved in a centralized way, we use the min-sum Message-Passing algorithm to solve it with a distributed process.

First, the factor graph corresponding to a given D2D pairs location is determined. For each D2D pair $i$, its interfering set $\mathcal{S}_{i}$ is defined according to the interference threshold $D_{0}$ and the inclusive neighbor set $\mathcal{A}_{i}$ is deduced. Then the factor graph is composed of $K$ variable nodes and $K$ factor nodes. An edge is joining factor node $M_{i}$ when variable node $P_{k}$ is $k$ belongs to $\mathcal{A}_{i}$.

\section{B. Joint transmit and receive antenna selection}

The first proposed algorithm aims at solving problem (8) by considering the transmit antenna selection and receive antenna selection together as joint optimization variables. In this case, the possible antenna selection matrix choices contain the permutation and combination of all the transmit and receive antenna selection possibilities.

The optimization problem is written as:

$$
\min _{\left[\left(\mathbf{V}_{1}, \mathbf{W}_{1}\right), \ldots,\left(\mathbf{V}_{K}, \mathbf{W}_{K}\right)\right] \in \mathcal{V} \times \mathcal{W}} \sum_{j=1}^{K}\left(-R_{i}\right)
$$

where $R_{i}$ is the data rate of user $i$, given by eq. (1). The objective is to determine, for each D2D pair $i$, the best pair of receive and transmit matrices, $\left(\mathbf{V}_{i}, \mathbf{W}_{i}\right)$. The min-sum MPA is then used, where the factor node $M_{i}$ corresponds to the function that computes the opposite of the data rate of D2D pair $i$, and the variable node $P_{i}$ corresponds to the value of $\left(\mathbf{V}_{i}, \mathbf{W}_{i}\right)$. The connections in the factor graph depend on the interfering set $\mathcal{S}_{i}$, as explained in the example of Fig.1. Then the MPA is used, as explained in section II-B.

The complexity of the MPA can be reduced. For instance, if $N_{t}=N_{r}=3$ and $N_{s}=2$, due to the sizes of sets $\mathcal{V}$ and $\mathcal{W}$, a specific node has $3 \times 3$ different combinations of possible transmit and receive antenna matrices. Assume a factor node is associated with $L$ variable nodes. If we simply consider all the combination results of these nodes' antenna selection possibilities, we will have a large number $9^{L}$ of computations to do in order to obtain the list of $M_{i}\left(P_{A_{i}}\right)$.

However, the receive antenna selection matrices of the interfering pairs $k \in \mathcal{S}_{i}$ do not have any influence on the data rate of user $i$, and consequently on $M_{i}$, as shown by eq. (1). In $M_{i}\left(P_{A_{i}}\right)$, when a pair of transmit and receive matrices is selected out of all the possibility of transmit and receive matrices for a pair of devices, the corresponding variable node containing the local parameters is fixed. The other associated variable nodes $P_{L_{i}}$ has an impact on that factor node. Because the receive matrix of variable $P_{L_{i}}$ have no influence on the value of $M_{i}\left(P_{A_{i}}\right)$, each interfering node has only three different impacts on the value of $M_{i}\left(P_{A_{i}}\right)$. That variable node itself can take only 3 different values. Consequently, for a single factor node, the required number of computations is limited to $9 \times 3^{L-1}$ for calculating $M_{i}\left(P_{A_{i}}\right)$.

To conclude, the min-sum MPA can be simplified by calculating the local performance matrices $M_{i}\left(P_{A_{i}}\right)$ first, taking into account the redundancy of all the possibilities for the combination of transmit and receive antenna selections. Then the algorithm is applied as explained in section II-B. The data rates are finally computed by taking into account the final values of $\left[\left(\mathbf{V}_{1}, \mathbf{W}_{1}\right), \ldots,\left(\mathbf{V}_{K},, \mathbf{W}_{K}\right)\right]$., Since the factor-graph is not cycle-free, the MPA does not necessarily converge. However, numerical simulation results have shown that high sum data rates can be achieved with only three iterations of the MPA. 


\section{Iterative transmit and receive antenna selection}

The joint transmit and receive antenna selection solution of Section III-B is jointly determining both parameters $\mathbf{V}$ and $\mathbf{W}$, by solving problem (9) with the min-sum MPA. Another possibility for solving the original problem (8) is to separate both transmit antenna selection and receive antenna selection problems.

The proposed algorithm iteratively solves the following optimization problems:

First, for fixed values of $\left[\mathbf{W}_{1}, \ldots, \mathbf{W}_{K}\right] \in \mathcal{W}$, solve:

$$
\min _{\left[\mathbf{V}_{1}, \ldots, \mathbf{V}_{K}\right] \in \mathcal{V}} \sum_{j=1}^{K}\left(-R_{i}\right)
$$

And then, for the fixed values of $\left[\mathbf{V}_{1}, \ldots, \mathbf{V}_{K}\right] \in \mathcal{V}$ obtained by solving (10), solve:

$$
\min _{\left[\mathbf{W}_{1}, \ldots, \mathbf{W}_{K}\right] \in \mathcal{W}} \sum_{j=1}^{K}\left(-R_{i}\right)
$$

The iterative algorithm was used in [4], but for point-to-point communications without multi-user interference.

This algorithm still relies on the min-sum MPA for transmit antenna selection. If we start with problem (10), then the initial values of the receive antenna matrices are randomly selected. The transmit antenna matrices are optimized with the MPA. The obtained transmit antenna selection matrices can be used in the next step in order to determine the receive antenna selection, with these fixed transmit antenna matrices. The receive antenna matrices are then optimized by exhaustive search, independently for each D2D pair, since $M_{i}$ only depends on $\mathbf{W}_{i}$ and not on the interferers receive matrices. These new receive antenna matrices are then used into the transmit antenna selection calculation at the next iteration.

The iterative algorithm leads to a solution for both transmit and receive antenna selection. Even though this solution is sub-optimal, simulation results will show that the obtained performance is still quite good. We refer to this algorithm as the iterative antenna selection algorithm.

To sum up, the specific procedure for this iterative algorithm is the following:

1) Randomly initialize the receive antenna selection at iteration index $x=0$. Set a maximum iteration index $x_{\max }$.

2) Solve problem (10) with the min-sum MPA, with fixed values of receive antenna matrices obtained for iteration $x$.

3) Solve problem (11) with exhaustive search, independenlty at each receiver, with fixed values of transmit antenna matrices obtained for iteration $x$. Increase iteration index to $x=x+1$.

4) If $x \leq x_{\max }$, go back to step 2) and repeat doing steps 2) and 3) until the iteration index $x$ reaches the maximum iteration index $x_{\max }$.

In this algorithm, the highest sum data rate is kept during all 2)-3) iterations, along with the corresponding transmit and receive matrices.

\section{Simulation Results}

The simulation assumptions are the following: the number of transmit and receive antennas is $N_{t}=N_{r}=3$ and $N_{s}=2$ antennas are selected. Thermal noise $N_{0}$ is white additive Gaussian with power spectral density $-174 \mathrm{dBm} / \mathrm{Hz}$. The channel bandwidth is that of a Resource Block, $B=180 \mathrm{KHz}$. Shadowing follows a log-normal distribution with standard deviation equal to $4 \mathrm{~dB}$. The power per D2D transmitter is $10 \mathrm{dBm}$. The D2D transmitters are uniformly distributed in a small cellular area of radius $r=0.2 \mathrm{~km}$. Each D2D receiver is uniformly located within $r_{0}=5$ to $r_{D}=50$ $\mathrm{m}$ from its transmitter. The interference distance is set to $D_{0}=0.25 \mathrm{~km}$. The path loss model is small cell's path loss: $L_{\mathrm{dB}}=140+36.8 \log _{10}(d)$, where $d$ is expressed in $\mathrm{km}$. MIMO channels are fully uncorrelated and follow a Rayleigh i.i.d. distribution.

The proposed joint and iterative antenna selection algorithms are referred to as Joint MPA and iterative MPA on the figures. They are compared with and individual optimization where each D2D pair $k$ exhaustively determines the best values for $\mathbf{W}$ and $\mathbf{V}$, without taking into account interference. Then their data rate is computed with the actual interference. It is also compared with a random allocation, where each transmit and receive matrix is randomly chosen.

Fig. 2 shows the Cumulative Distribution Function (CDF) of the sum data rate when $K=5$, with joint MPA algorithm, iterative MPA, individual optimization and random allocation. The joint MPA algorithm provides larger sum data rates than the other algorithms. Joint MPA, iterative MPA and individual optimization are far more efficient than random allocation, that does not benefit from MIMO space diversity to select the best individual streams, the least interfered ones or a combination of both criteria. Individual optimization only selects the best individual streams, neglecting interference. It is consequently less efficient than joint and iterative MPA, but the achieved rate is still high, since the interference is quite low in the studied scenario with $K=5$. Finally, the iterative MPA provides a good trade-off between performance and complexity, compared to the joint MPA.

Fig. 3 shows the average sum data rate when $K$ varies from 3 to 5 with all four algorithms. Similarly to the previous figure, joint MPA outperforms the other algorithms, but iterative MPA is still quite efficient. The average sum rate is at least $19 \%$ larger with joint MPA than with random allocation.

Finally, Fig. 4 represents the fairness index among D2D pairs, defined as follows:

$$
F I=\frac{\left(\sum_{i=1}^{K} R_{i}\right)^{2}}{K \sum_{i=1}^{K}\left(R_{i}\right)^{2}}
$$

We can see that the MPA are more fair than individual optimization and random allocation, because they take into account interference. The proposed algorithms consequently avoid situations where one D2D receiver would receive large interference power and thus obtain far lower rates than the others. Consequently, the simulation results show that both 


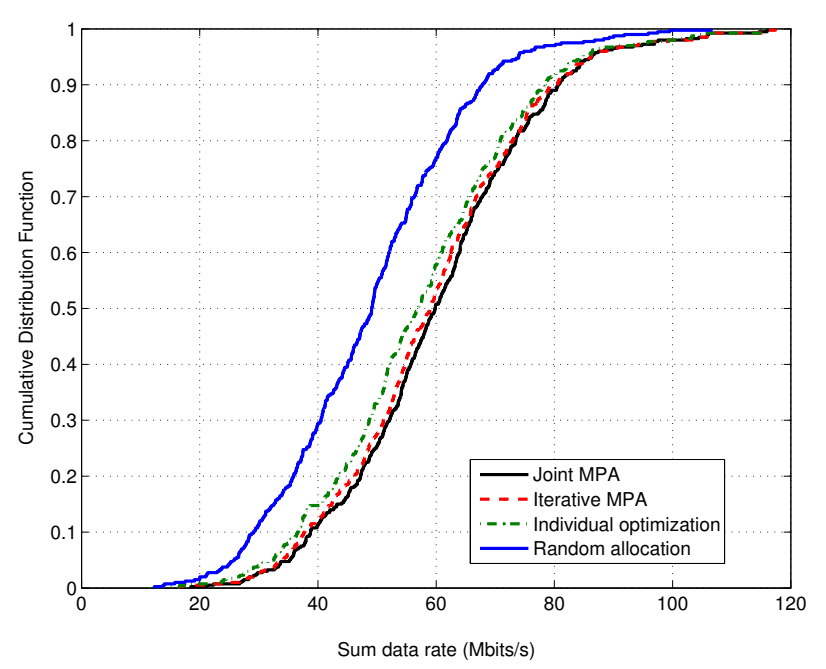

Fig. 2. $\mathrm{CDF}$ of the sum data rate when $K=5$

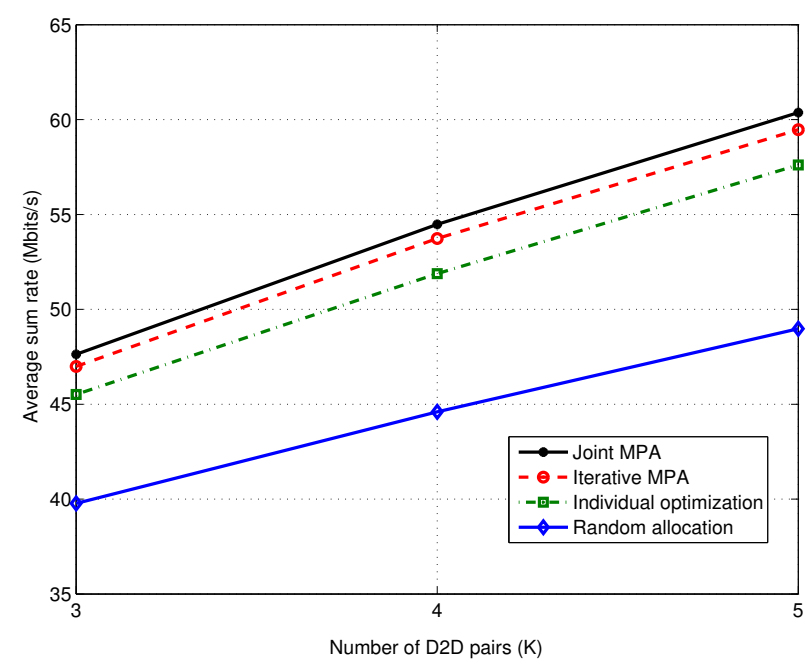

Fig. 3. Average sum rate as a function of $K$

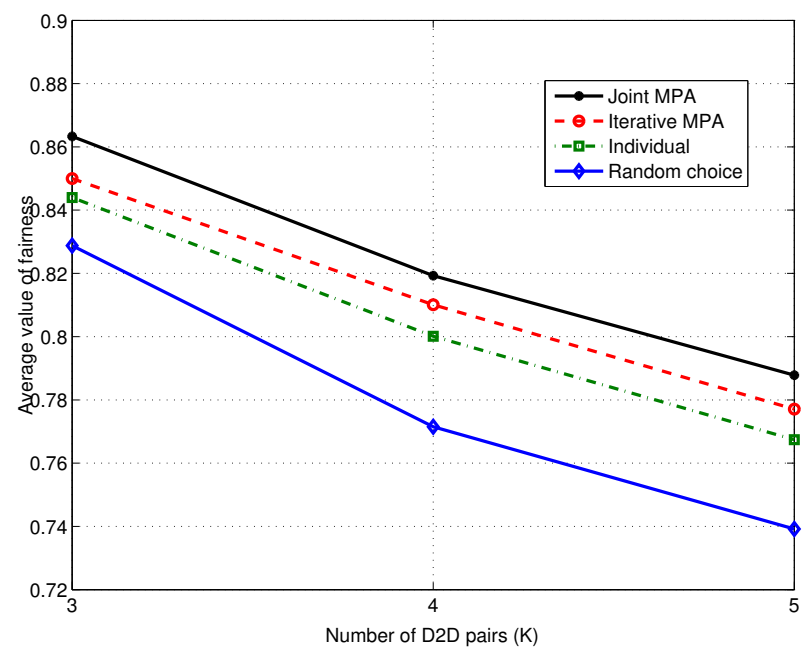

Fig. 4. Average fairness as a function of $K$
MPA not only increase the sum rate, but also increase the fairness.

\section{CONCLUSIONS}

This paper has proposed two distributed algorithms based on the min-sum Message-Passing algorithm for determining the best sets of transmit and receive antenna selection matrices in MIMO D2D communications where all D2D pairs share the same channel. The first algorithm jointly optimizes both transmit and receive antenna selection matrices, while the second one iteratively optimizes transmit, and then receive antenna selection matrices. Both algorithms perform well in terms of achieved rates and fairness. Thanks to the minsum MPA, a distributed implementation of these algorithms is feasible, which is particularly interesting for future 5G D2D communications.

\section{ACKNOWLEDGEMENT}

This work was partially funded through French National Research Agency (ANR) project ACCENT5 with grant agreement code: ANR-14-CE28-0026-02.

\section{REFERENCES}

[1] M. N. Tehrani, M. Uysal and H. Yanikomeroglu, Device-to-device communication in $5 G$ cellular networks: challenges, solutions, and future directions, IEEE Communications Magazine, 52(5), May 2014

[2] M. Pischella, R. Zakaria and D. Le Ruyet, Resource Block level power allocation in asynchronous multi-carrier D2D communications , IEEE Communication Letters, 21(4), 813-816, Apr. 2017

[3] A.S. Molish and M.Z. Win, MIMO systems with antenna selection, IEEE Microwave Magazine, 5(1), March 2004.

[4] A. Gorokhov, M. Collados, D. Gore and A. Paulraj, Transmit/receive MIMO antenna subset selection, Proc. IEEE International Conference on Acoustics, Speech, and Signal Processing, pp.13-16, May 2004.

[5] F. Kschischang, B. J. Frey and H. A. Loeliger Factor graphs and the sum-product algorithm, IEEE Transactions on information theory, 47(2), 498-519, feb. 2001

[6] S. Aji and R. McEliece, The generalized distributive law, IEEE Transactions on Information Theory, vol. 46, no. 2, pp. 325 343, march 2000.

[7] J. S. Yedidia, Message-passing algorithms for inference and optimization, Journal of Statistical Physics, vol. 145, no. 4, pp. 860890, Nov.2011.

[8] I. Sohn, S. H. Lee, and J. Andrews, Belief propagation for distributed downlink beamforming in cooperative mimo cellular networks, IEEE Transactions on Wireless Communications, vol. 10, no. 12, pp. 4140 4149, dec. 2011.

[9] B. L. Ng, J. Evans, S. Hanly, and D. Aktas, Distributed downlink beamforming with cooperative base stations, IEEE Transactions on Information Theory, vol. 54, no. 12, pp. 5491 5499, dec. 2008.

[10] I. M. Guerreiro, D. Hui, and C. C. Cavalcante, A distributed approach to precoder selection using factor graphs for wireless communication networks, EURASIP Journal on Applied Signal Processing, vol. 83, 2013.

[11] I. M. Guerreiro, C. C. Cavalcante, D. Hui Adaptation with reduced-size message pass to precoder selection in multi-cell MIMO systems, IEEE Sensor Array and Multichannel Signal Processing Workshop (SAM), pp. 1-4, July 2016

[12] M.Guillaud, M. Rezaee, G. Matz, G. Interference alignment via message-passing, IEEE International Conference on Communications (ICC), pp. 5741-5746, June 2014 